\title{
Prognostic factors for early relapse in non-metastatic triple negative breast cancer - real world data
}

\author{
Rita Félix Soares, Ana Rita Garcia, Ana Raquel Monteiro, Filipa Macedo, Tatiana Cunha Pereira, \\ Joana Cunha Carvalho, António Pêgo, Mónica Mariano, Pedro Madeira, Sara Póvoa, Sofia Broco, \\ Teresa Carvalho, Isabel Pazos, Gabriela Sousa \\ Portuguese Oncology Institute of Coimbra Francisco Gentil, Coimbra, Portugal
}

\begin{abstract}
Background: Triple negative breast cancer (TNBC) has the worst prognosis amongst all subtypes. Studies have shown that the achievement of pathologic complete response in the breast and axilla correlates with improved survival. The aim of this study was to identify clinical or pathological features of real-life TNBC patients with a higher risk of early relapse.

Materials and methods: Single-centre retrospective analysis of 127 women with TNBC, stage II-III, submitted to neoadjuvant treatment and surgery between January 2016 and 2020. Multivariate Cox regression analysis for disease free survival (DFS) at 2 years was performed and statistically significant variables were computed into a prognostic model for early relapse.

Results: After 29 months of median follow-up, 105 patients (82.7\%) were alive and, in total, 38 patients (29.9\%) experienced recurrence. The 2-year DFS was $73 \%$ (95\% Cl: 21.3-22.7). In multivariate analysis, being submitted to neoadjuvant radiotherapy [HR 2.8 (95\% Cl: 1.2-6.4), $\mathrm{p}=0.017$ ] and not achieving pathologic complete response [HR 0.3 (95\% Cl: $0.1-1.7), \mathrm{p}=0.011$ ] were associated with higher risk of recurrence. In our prognostic model, the presence of at least one of these variables defined a subgroup of patients with a worse 2-year DFS than those without these features ( $59 \%$ vs. 90\%, $p<0.001$, respectively).

Conclusions: In this real-life non-metastatic TNBC cohort, neoadjuvant radiotherapy (performed due to insufficient clinical response to neoadjuvant chemotherapy or significant toxicity) impacted as an independent prognostic factor for relapse along with the absence of pathologic complete response identifying a subgroup of higher risk patients for early relapse that might merit a closer follow-up.
\end{abstract}

Key words: triple negative breast cancer; prognostic factors; early relapse; survival Rep Pract Oncol Radiother 2021;26(4):563-572

\section{Introduction}

Triple begative breast cancer (TNBC) accounts approximately for 15 percent of breast cancers diagnosed worldwide, which accounts for almost 200,000 cases each year [1]. In Portugal, in 2018, the number of new diagnosed cases of breast cancer was $6,974[2]$. TNBC is defined as: $\leq 1 \%$ expression of estrogen receptor (ER) and progesterone receptor (PR) by immunohistochemistry (IHC), and human epidermal growth factor receptor 2 (HER2) that are either 0 to $1+$ by IHC, or IHC $2+$ and fluorescence in situ hybridization (FISH)-negative (not amplified), according to the American Society of Clinical Oncology/College of American Pathologists (ASCO/CAP) guidelines [3, 4].

Address for correspondence: Rita Félix Soares, Portuguese Oncology Institute of Coimbra Francisco Gentil, Avenida Bissaya Barreto no. 98 , 3000-075 Coimbra, Portugal; e-mail: r.felixsoares@gmail.com 
The majority of TNBCs are histologically defined as non-specific invasive breast cancer (approximately $95 \%$ ) and the other $5 \%$ are infiltrating lobular carcinoma, secretory cancer, medullary carcinoma, adenoid cystic carcinoma and fibromatosis-like spindle-cell metaplastic carcinoma [5]. This small proportion of TNBCs have a low aggressive potential and follow an indolent course contrasting with the invasive ductal carcinomas which are associated with high rates of early distant recurrence and short survival times compared to other breast cancer subtypes [6-12].

Unlike other breast cancer subtypes, there are no approved targeted treatments available, although immunotherapy (in combination with chemotherapy) is available for those with advanced TNBC that expresses programmed cell death ligand 1 . Therefore, chemotherapy is still the standard of care for early and late stages of TNBC patients. Many studies have demonstrated the benefit of chemotherapy in neoadjuvant, adjuvant and metastatic setting in TNBC. Neoadjuvant chemotherapy (NACT) is the preferred treatment approach to potentially increase the likelihood of tumor resectability and breast conservation. TNBC tends to behave more aggressively being associated with the worst prognosis amongst all breast cancer subtypes. The risk of distant recurrence and death peaks at approximately three years after diagnosis and declines rapidly thereafter [13]. During this period, TNBC is characterized by higher relapse rates compared with hormone receptor positive breast cancers, although the latter tends to continue to recur for decades. Patients with TNBC have a poorer short-term (first five to seven years) prognosis compared with patients with other breast cancer subtypes $[3,6]$.

A subgroup of TNBCs is inherently chemosensitive and achieves a pathological complete response (pCR) following combination anthracycline/taxane-based NACT [8-14]. These patients who achieve pCR after NACT have a higher rate of survival compared to those with residual disease [6, 14]. The basis for this variation in response to chemotherapy and survival remains unclear. In general, in breast cancer, tumor size is correlated with nodal involvement, and the prognostic value of the two factors is independent of each other. Nevertheless, in TNBC, the correlation between tumor size and lymph node status may not be linear since even the small tumors may have a high rate of node positivity [15]. In fact, in TNBC, the nodal status has been described as an independent prognostic factor [16].

The aim of our retrospective study is to analyse the clinicopathological characteristics and prognostic significance of putative prognostic factors in TNBC as well as to determine short-term outcomes in these patients at our institution.

\section{Materials and methods}

\section{Study design and data source}

This is single-centre retrospective analysis of 127 women with non-metastatic TNBC. Data regarding patient characteristics, tumor pathological characteristics, analytical workup, treatment data, toxicities and outcomes were reviewed and analyzed retrospectively from the patient's electronic medical records from the Portuguese Oncology Institute of Coimbra Francisco Gentil. The study was reviewed and approved by the Ethics Committee of the Portuguese Oncology Institute of Coimbra Francisco Gentil and a waiver of written informed consent from patients was granted considering the retrospective nature of the study.

\section{Patients eligibility}

Eligible patients were $\geq 18$ years old, diagnosed with non-metastatic TNBC treated between January 2016 and January 2020. All patients underwent NACT and/or NART and surgery (breast conserving surgery or modified radical mastectomy). Patients with metastatic presentation, patients without histologically documentation of TNBC, patients who underwent upfront breast surgery and patients not eligible for neoadjuvant treatment were excluded.

\section{Definitions}

Breast tumors with IHC-negative ER and PR associated with HER-2 score 0 or 1 with IHC staining or FISH negative expression were defined as TNBC. Tumors were staged according to guidelines [17]. A pCR was defined as no residual invasive disease in the breast, with or without residual in situ disease, and no residual disease in the axillary lymph nodes (ypT0/is ypN0). Disease free survival (DFS) was defined as the time from the beginning of neoadjuvant treatment until disease recurrence detection. Overall survival (OS) was defined as the time 
from diagnosis to death or last follow-up. Toxicities were graded according to CTCAE version 5 .

\section{Statistical analysis}

Chi-square tests and analysis of variance (ANOVA) or Kruskal-Wallis tests were used to assess differences in categorical and continuous variables. Categorical variables were described in percentages. Continuous variables were characterized by median and specific interval. For some continuous variables, like tumor markers CEA and CA 15.3, neutrophil/lymphocyte ratio, platelet/lymphocyte ratio and Ki-67, the cut-off used for the Cox regression analysis was determined by ROC curve and determination of the Youden Index. CEA and CA 15.3 ratios were calculated by dividing the value before the beginning of neoadjuvant treatment by the value after surgery. Neutrophil/lymphocyte ratio was calculated by dividing the number of neutrophils by the number of lymphocytes from the complete blood count before the start of neoadjuvant treatment, and platelet/lymphocyte ratio was calculated by dividing the number of platelets by the number of lymphocytes from the complete blood count before the start of neoadjuvant treatment.

A univariate logistic regression analysis was used to estimate the associations between demographic, clinicopathologic, tumor and treatment characteristics with survival outcomes (DFS and OS at 24 months). The characteristics were selected based on statistical significance which was set at $\mathrm{p}<0.05$. All the characteristics considered significant in the univariate analysis were included in the multivariate Cox regression analysis. We report hazard ratios (HR) and 95\% confidence intervals (CI) with $\mathrm{p}<0.05$ considered significant. Based on the results of the multivariate analysis for DFS, a risk model was created. Survival plots were generated with the Kaplan-Meier method with log-rank $\mathrm{p}<0.05$ defined as significant. All analyses were conducted using the Statistical Package for Social Sciences (SPSS v26.0, SPSS Inc., Chicago, IL, USA) software.

\section{Results}

\section{Patient characteristics}

From January 2016 through January 2020, 127 patients were analyzed. The baseline demographic, disease and tumor characteristics are represented in Table 1. Briefly, the median age was 52 years [in-
Table 1. Patient and tumor characteristics

\begin{tabular}{|c|c|}
\hline & No. (\%) \\
\hline Age (y), median [range] & $52[26-74]$ \\
\hline Female gender & $127(100)$ \\
\hline \multicolumn{2}{|l|}{ ECOG PS } \\
\hline 0 & $104(81.9)$ \\
\hline 1 & $20(15.7)$ \\
\hline 2 & $3(2.4)$ \\
\hline \multicolumn{2}{|l|}{ BRCA mutation status } \\
\hline Present & $6(4.7)$ \\
\hline Undetermined clinical significance mutation & $5(3.9)$ \\
\hline Unknown & $39(30.7)$ \\
\hline Negative & $77(60.6)$ \\
\hline Premenopausal & $43(33.9)$ \\
\hline \multicolumn{2}{|l|}{ Histologic subtype } \\
\hline Ductal invasive carcinoma & $110(86.6)$ \\
\hline Apocrine carcinoma & $8(6.3)$ \\
\hline Metaplastic carcinoma & $4(3.1)$ \\
\hline Other & $5(3.9)$ \\
\hline \multicolumn{2}{|l|}{ Grade } \\
\hline 1 & $8(6.3)$ \\
\hline 2 & $61(48)$ \\
\hline 3 & $57(44.9)$ \\
\hline Unknown & $1(0.8)$ \\
\hline \multicolumn{2}{|l|}{ Ki-67 } \\
\hline$\geq 70 \%$ & $73(57.5)$ \\
\hline$<70 \%$ & $50(39.4)$ \\
\hline Unknown & $4(3.1)$ \\
\hline \multicolumn{2}{|l|}{ ст } \\
\hline 0 & $2(1.6)$ \\
\hline 2 & $52(41)$ \\
\hline 3 & $61(48)$ \\
\hline 4 & $12(9.4)$ \\
\hline \multicolumn{2}{|l|}{$\mathrm{cN}$} \\
\hline 0 & $51(40.2)$ \\
\hline 1 & $68(53.5)$ \\
\hline 2 & $8(6.3)$ \\
\hline \multicolumn{2}{|l|}{ Clinical stage } \\
\hline$\| A$ & $3(2.4)$ \\
\hline IIB & $31(24.4)$ \\
\hline IIIB & $62(48.8)$ \\
\hline IIIC & $31(24.4)$ \\
\hline \multicolumn{2}{|l|}{ Neutrophil/lymphocyte ratio } \\
\hline$\geq 1.8$ & $74(58.3)$ \\
\hline$<1.8$ & $53(41.7)$ \\
\hline \multicolumn{2}{|l|}{ Platelet/lymphocyte ratio } \\
\hline$\geq 0.1 \times 10^{3}$ & $59(46.5)$ \\
\hline$<0.1 \times 10^{3}$ & $68(53.5)$ \\
\hline
\end{tabular}

ECOG PS - Eastern Cooperative Oncology Group performance status; $y$-years; no. - number 
terval 26-74 years], the majority of patients had an Eastern Cooperative Oncology Group performance status (ECOG PS) of 0, 34\% were premenopausal and only $5 \%$ were BRCA mutated. Concerning the histologic subtype, the majority of patients had ductal invasive carcinoma, 93\% had Modified Bloom Richardson (MBR) pathologic grade 2 or 3 and $58 \%$ had Ki67 $\geq 70 \%$. The majority of patients had a clinical T status $\geq 3$, clinical $N$ status $\geq 1$ and $73 \%$ had stage III disease according to the $8^{\text {th }}$ edition of the American Joint Committee on Cancer (AJCC) classification. The pre-treatment neutrophil/lymphocyte ratio was $\geq 1.80$ in $58 \%$ of patients and the platelet/lymphocyte ratio was $\geq 0.1 \times 10^{3}$ in $47 \%$.

\section{Treatment, toxicities and response}

All patients received neoadjuvant chemotherapy: $97.6 \%$ anthracycline and taxane-based chemotherapy, $0.8 \%$ taxane-based (due to cardiac comorbidities) and $1.6 \%$ other regimens (taxane and platinum regimens). One patient underwent taxane and platinum regimen due to a concomitant primary ovarian cancer and another patient underwent anthracycline regimen and did not proceed to the taxane treatment due to hematologic toxicity grade 4 (Tab. 2).

The CEA ratio was $\geq 0.66$ in $75.6 \%$ and the CA 15.3 ratio was $\geq 0.8$ in and $89.8 \%$.

In $13 \%$ of the patients, neoadjuvant radiotherapy was performed due to clinically progressive disease and/or grade $3 / 4$ adverse events. Of the 16 patients that underwent NART, none achieved pCR. In fact, only 5 patients experienced downstaging of the disease with neoadjuvant treatment at pathologic analysis of the specimen. Of the 16 patients, 6 had pathologic node positive disease and $3 \mathrm{had} \geq \mathrm{pT} 3$ disease.

The totality of patients underwent breast surgery: radical mastectomy in $95.3 \%$ and in $4.7 \%$ breast conserving surgery.

Adjuvant radiotherapy was performed in 59.8\% and $7.9 \%$ underwent adjuvant chemotherapy with capecitabine.

Only one patient underwent 8 cycles of adjuvant chemotherapy with capecitabine. The other patients discontinued treatment due to grade $3 / 4$ adverse events ( 5 patients had hematologic toxicity, 1 patient had gastrointestinal toxicity, 3 patients had hand-foot syndrome and 1 patient had Stevens-Johnson syndrome).
Table 2. Treatment and pathologic characteristics

\begin{tabular}{|c|c|}
\hline Treatment characteristics & No. (\%) \\
\hline \multicolumn{2}{|l|}{ Neoadjuvant chemotherapy } \\
\hline Anthracycline and taxane-based & $124(97.6)$ \\
\hline Taxane-based only & $1(0.8)$ \\
\hline Other & $2(1.6)$ \\
\hline \multicolumn{2}{|c|}{ CEA pre-treatment/after surgery ratio } \\
\hline$\geq 0.66$ & $96(75.6)$ \\
\hline$<0.66$ & $31(24.4)$ \\
\hline \multicolumn{2}{|c|}{ CA 15.3 pre-treatment/after surgery ratio } \\
\hline$\geq 0.8$ & $114(89.8)$ \\
\hline$<0.8$ & $13(10.2)$ \\
\hline Neoadjuvant radiotherapy & $16(12.6)$ \\
\hline \multicolumn{2}{|l|}{ Type of breast surgery } \\
\hline Radical mastectomy & $121(95.3)$ \\
\hline Breast conserving surgery & $6(4.7)$ \\
\hline Adjuvant radiotherapy & $76(59.8)$ \\
\hline Adjuvant chemotherapy & $10(7.9)$ \\
\hline \multicolumn{2}{|l|}{ Pathologic characteristics } \\
\hline \multicolumn{2}{|l|}{ Cellularity } \\
\hline $0-5 \%$ & $58(45.6)$ \\
\hline $10-50 \%$ & $33(26)$ \\
\hline$>50 \%$ & $5(4)$ \\
\hline Unknown & $31(24.4)$ \\
\hline \multicolumn{2}{|l|}{ Lymph nodes removed in surgery } \\
\hline$<10$ & $13(15.3)$ \\
\hline$\geq 10$ & $72(84.7)$ \\
\hline
\end{tabular}

\begin{tabular}{|c|c|}
\hline \multicolumn{2}{|c|}{ Pathology tumor stage [pT] } \\
\hline$<$ pT3 & $121(95.3)$ \\
\hline$\geq$ pT3 & $6(4.7)$ \\
\hline \multicolumn{2}{|l|}{ Pathology nodal stage [pN] } \\
\hline pNO & $100(79)$ \\
\hline $\mathrm{pN}+$ & $27(21)$ \\
\hline $\mathrm{pCR}$ & $62(48.8)$ \\
\hline Lymphovascular invasion & $19(15)$ \\
\hline Lymphatic emboli & $9(7.1)$ \\
\hline Deep margin involvement & $5(3.9)$ \\
\hline Calcification & $2(1.6)$ \\
\hline
\end{tabular}

no - number; $\mathrm{pCR}$ - pathologic complete response

The tumor pathologic characteristics are described in Table 2. The pCR was $48.8 \%$, with $66.9 \%$ being submitted to axillary lymph node dissection. In $21 \%$ of patients the pathologic $\mathrm{N}$ status was $\geq 1$. Lymphovascular invasion was detected in $15 \%$, lymphatic emboli in $7.1 \%$, deep margin 
Table 3. Adverse events with neoadjuvant chemotherapy

\begin{tabular}{|c|c|c|}
\hline Event & $\begin{array}{c}\text { Grade 1-2 } \\
\text { No. (\%) }\end{array}$ & $\begin{array}{c}\text { Grade 3-4 } \\
\text { No. (\%) }\end{array}$ \\
\hline \multicolumn{3}{|l|}{ Hematologic } \\
\hline Anemia & $62(48.8 \%)$ & $7(5.5 \%)$ \\
\hline Neutropenia & $17(13.4 \%)$ & $13(10.2 \%)$ \\
\hline \multicolumn{3}{|l|}{ Gastrointestinal } \\
\hline Nausea & $41(32.3 \%)$ & $5(3.9 \%)$ \\
\hline Mucositis & $35(27.6 \%)$ & $5(3.9 \%)$ \\
\hline Constipation & $12(9.4 \%)$ & 0 \\
\hline Dysgeusia & $6(4.7 \%)$ & 0 \\
\hline Diarrhea & $5(3.9 \%)$ & $1(0.8 \%)$ \\
\hline Vomiting & $4(3.1 \%)$ & 0 \\
\hline Anorexia & $2(1.6 \%)$ & $1(0.8 \%)$ \\
\hline Hepatitis & $1(0.8 \%)$ & $1(0.8 \%)$ \\
\hline Neurotoxicity & $25(19.7 \%)$ & $2(1.6 \%)$ \\
\hline \multicolumn{3}{|l|}{ Cutaneous } \\
\hline Alopecia & 127 (100\%) & NA \\
\hline Onycholysis & $12(9.4 \%)$ & $3(2.4 \%)$ \\
\hline Hand-foot syndrome & $5(3.9 \%)$ & $1(0.8 \%)$ \\
\hline Rash & $4(3.1 \%)$ & $2(1.6 \%)$ \\
\hline Fatigue & $16(12.6 \%)$ & $1(0.8 \%)$ \\
\hline Myalgias & $11(8.7 \%)$ & 0 \\
\hline \multicolumn{3}{|l|}{ Ocular } \\
\hline Watering eyes & $3(2.4 \%)$ & 0 \\
\hline Dry eye & $2(1.6 \%)$ & 0 \\
\hline Pyrexia & $1(0.8 \%)$ & 0 \\
\hline Hypersensitivity reaction & $1(0.8 \%)$ & $2(1.6 \%)$ \\
\hline
\end{tabular}

No. - number; NA — not applicable

involvement in $3.9 \%$ and calcifications in $1.6 \%$. In $30 \%$ pathologic celularity was $\geq 10 \%$.

The safety profile of the different neoadjuvant regimens was consistent with the known adverse events of each agent, which are represented in Table 3 . The main toxicities observed were grade 1-2. The most common grade 3 or 4 adverse event was neutropenia. Discontinuation of neoadjuvant treatment due to grade $3 / 4$ toxicities was observed in 17 patients, of which 3 discontinued also due to clinically detected progressive disease.

Recurrence, overall survival and disease free survival

After 29 months of median follow-up (interval 6-82 months), 105 patients (82.7\%) were alive and in total 38 patients (29.9\%) experienced recurrence. Briefly, 14 patients recurred locoregionally with ganglionar, cutaneous and/or muscular involvement. After local biopsy, TNBC was histology confirmed in all 14 patients. Of these patients, 13 were submitted to radical mastectomy, 1 to breast conserving surgery and 8 underwent adjuvant radiotherapy. Of the 6 patients that did not go adjuvant radiotherapy, 3 had been submitted to neoadjuvant radiotherapy due to toxicity or clinical progression during NACT, 1 patient had an initial stage IIB and 2 patients had achieved pCR.

The most frequent distant sites of recurrence were lungs (16 patients), brain (10 patients), bone (9 patients) and liver (5 patients). One patient developed renal and peritoneal histologically confirmed breast cancer metastasis. The majority of patients had 1 or 2 involved sites. Only 6 patients had $\geq 3$ involved sites of metastatic disease. Of the 10 patients that developed brain metastasis, 2 were submitted to radiosurgery treatment, 5 whole-brain radiotherapy, 1 patient underwent brain surgery followed by radiosurgery and after local recurrence whole-brain radiotherapy. There were 2 patients that did not meet the functional status to treat brain metastasis and underwent exclusive symptomatic treatment.

After recurrence diagnosis, 24 patients underwent palliative chemotherapy treatment. The remaining patients underwent local treatments and/or exclusive symptomatic and supportive care after recurrence diagnosis. The totality of patients treated with palliative chemotherapy did not undergo more than 3 lines of therapy.

The 2-year DFS and OS was 73\% (95\% CI: 21.3-22.7) and 89\% (95\% CI: 23-23.7), respectively.

In univariate analysis for DFS, neoadjuvant radiotherapy (NART), pCR, BRCA mutation, pathologic node and $\mathrm{T}$ status and pathologic cellularity had significance. In multivariate analysis, being submitted to NART [HR: 2.8 (95\% CI: 1.2-6.4), $\mathrm{p}=0.017)$ and not achieving pCR [HR: $0.3(95 \%$ CI: 0.1-1.7), $\mathrm{p}=0.011]$ were associated with higher risk of recurrence (Tab. 4). In univariate analysis for OS, NART, pCR and pathologic lymphatic emboli had significance. In multivariate analysis, being submitted to NART [HR = 7.1 (95\% CI: 2.3-22.2), $\mathrm{p}=0.001]$ and the presence of pathologic lymphatic emboli [HR: 4.7 (95\% CI: 1.2-17.3), $\mathrm{p}=0.022$ ] negatively impacted OS (Tab. 4).

The presence of at least one of these variables (not achieving pCR and being submitted to NART) 


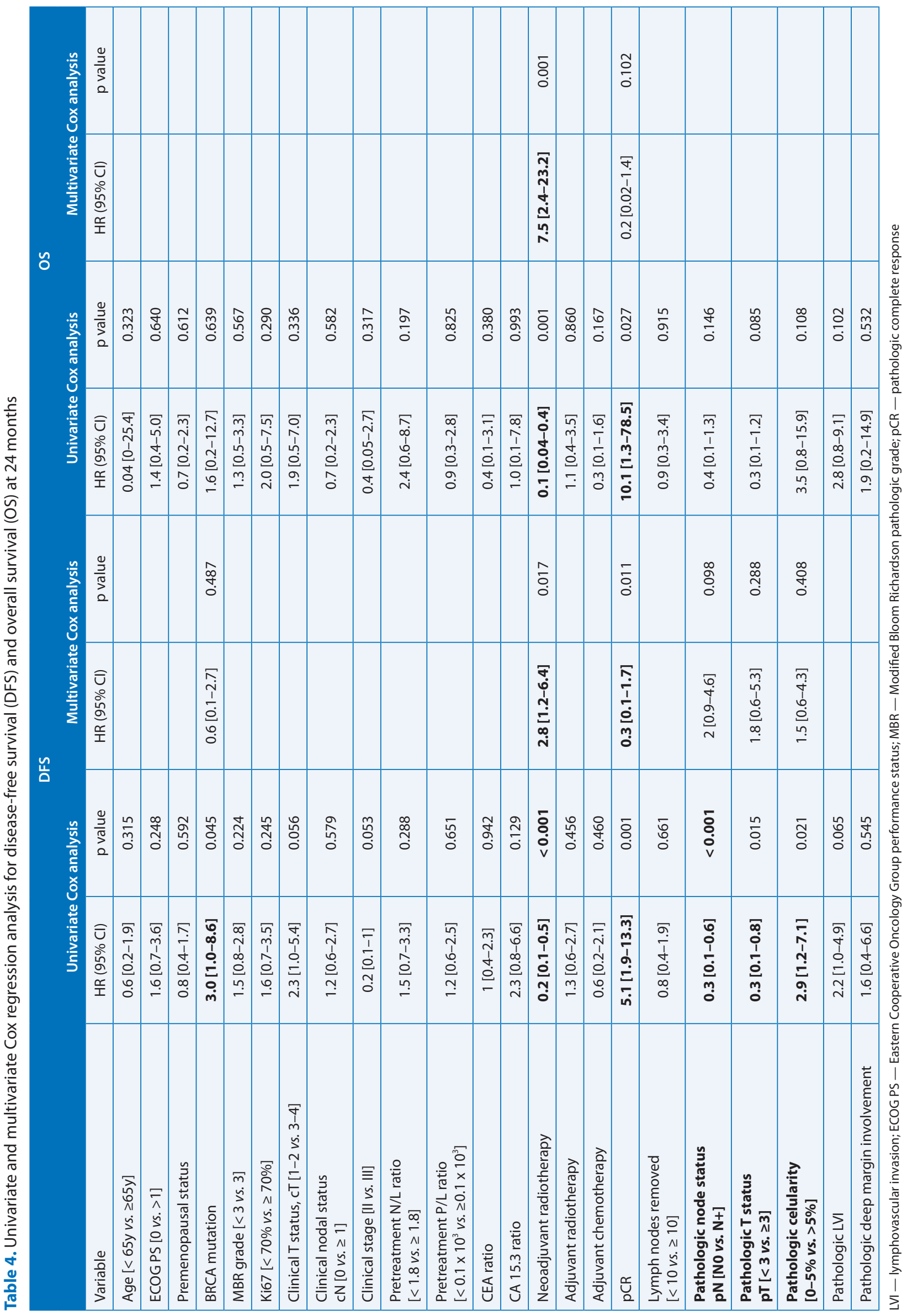




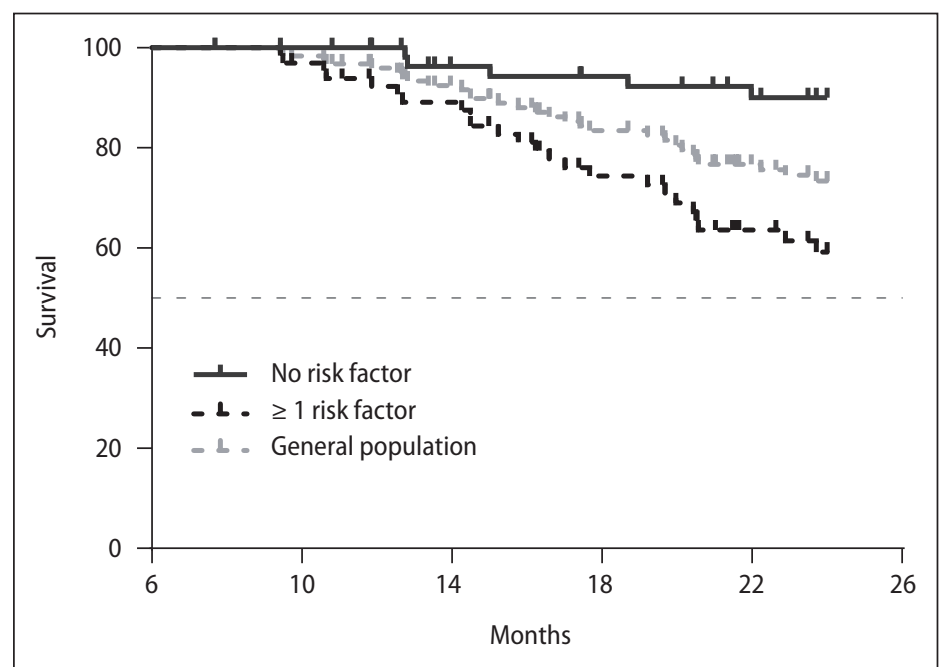

$\begin{array}{lccc} & \text { no (\%) } & \text { DFS 24 months } & \text { p value } \\ \text { General population } & 127(100 \%) & 73 \% & \\ \text { No risk factor } & 61(48 \%) & 90 \% & <0.001 \\ \text { Not achieving pCR and/or being submitted to NART } & 66(52 \%) & 59 \% & \end{array}$

Figure 1. Risk model for disease free survival at 24 months and Kaplan-Meier curve according to risk factors identified in the multivariate Cox regression

defined a subgroup of patients with a worse DFS at 24 months than those without these features (59\% vs. $90 \%, \mathrm{p}<0.001$, respectively). With this data, we developed a risk model that is represented in Figure 1.

\section{Discussion}

Despite the widespread acknowledgment of the poor clinical outcome of TNBC, the prognostic value of specific morphological and biological features of these tumors continues to raise a substantial degree of uncertainty and controversy.

This study represents a real-life cohort, where the majority of our TNBC patients had relatively large tumors (57.4\% with clinical $\mathrm{T} \geq 3$ ) and 59.8\% had positive axillary lymph nodes at presentation. The predominant subtype was invasive ductal carcinoma and $44.9 \%$ had poorly differentiated histology. In this study, the prognostic significance of putative well-established prognostic factors, such as age, menopausal status, clinical and pathologic nodal status, size of the tumor, grade, Ki-67, the presence of lymphovascular invasion, was assessed. Pathologic node and T status and pathologic cellularity were found as prognostic markers in the
DFS univariate analysis but lost independency in multivariate analysis. In multivariate analysis, only NART and the non-achievement of pCR impacted as independent prognostic factors for disease recurrence, identifying a subgroup of higher risk patients for early recurrence. Concerning OS, NART and the non-achievement of PCR were found as prognostic markers in the univariate analysis, although pCR lost its independency in multivariate analysis.

Among patients treated with neoadjuvant chemotherapy, achievement of a pCR is prognostically significant. The association between $\mathrm{pCR}$ and long-term outcome is stronger in patients with aggressive breast cancer subtypes, including TNBC [14]. Considering pCR, our results are in line with literature, since its non-achievement correlates with worst DFS. The role of $\mathrm{pCR}$ on patient outcomes has been demonstrated to be the highest in TNBC and HER2 positive breast cancer [17-19]. In 2014, the results of the Collaborative Trials in Neoadjuvant Breast Cancer (CTNeoBC) pooled analysis were published [14]. This study, funded by the United States Food and Drug Administration (FDA), included 12 clinical trials and 11,955 patients. The authors reported that patients who attained pCR defined as ypT0ypN0 or ypT0/isypN0 had better 
outcomes and that it was the greatest in aggressive tumor subtypes (in patients with TNBC, event free survival HR: $0.24,95 \%$ CI: $0.18-0.33$, and overall survival HR: 0.16, 95\% CI: 0.11-0.25). Following this report, pCR has been used by the FDA as an endpoint to facilitate accelerated approval for certain agents. In our study, the non-achievement of pCR was identified as an independent prognostic factor for DFS; however, it did not impact OS perhaps due to the relatively small cohort and short follow-up. Even though the pCR was $48 \%$, in our study, there was a significant percentage (66.9\%) of patients that were submitted to axillary lymph node dissection. For women who received neoadjuvant therapy, the approach to the axilla usually depends on the presence of suspicious nodes prior to neoadjuvant therapy and response to treatment. In our study there was a significant percentage of patients with large tumors and a node positive status. Per protocol, in our institution, patients with a node positive status undergo axillary lymph node dissection. Additionally, some patients with positive sentinel lymph node on surgery were also submitted to axillary lymph node dissection, justifying the significant percentage of lymph node dissection. Nevertheless, considering the comorbidities associated with axillary lymph node dissection, risk benefit of the procedure should be considered specially in patients with clinical N1 disease and a good response to neoadjuvant treatment.

In our study, NART was performed with the objective of downsizing inoperable tumors in patients who had progressive disease and/or grade 3/4 toxicities during neoadjuvant chemotherapy treatment. Radiotherapy has been clearly demonstrated to reduce locoregional and distant relapse after breast conserving surgery and radical mastectomy although its role as a neoadjuvant therapy is not well established in terms of outcomes. Neoadjuvant radiotherapy is indicated after chemotherapy if tumor resectability is not achieved. There are some studies that demonstrate its benefit as neoadjuvant treatment. A study of 134 patients with locally advanced breast cancer submitted to neoadjuvant radiotherapy (with no systemic treatment administered as neoadjuvant treatment) demonstrated a pCR in $15 \%$ of the patients, a relapse rate of $61.9 \%$, and a 5 -year disease free survival and overall survival of $39.2 \%$ and $55.1 \%$, respectively [20]. In this study the hormone receptor status did not influence the clinical response to neoadjuvant radiotherapy although non-significantly higher response rate was noticed in patients with hormone receptor negative compared to hormone receptor positive breast cancer [20]. However, there are few data about patients who are initially refractory to neoadjuvant chemotherapy and who are submitted to salvage radiotherapy. Coelho et al., conducted one of the largest retrospective studies of the implementation of NART in patients refractory to neoadjuvant chemotherapy and ineligible for surgical resection. The study demonstrated that radiotherapy could be an alternative to patients with unresectable tumors after neoadjuvant chemotherapy with overall survival advantage for patients with tumors resected after neoadjuvant salvage radiotherapy compared with those who were unresected. However, almost half of the patients were hormone receptor negative and this subset of patients had lower responses to neoadjuvant radiotherapy compared with hormone receptor positive patients [21].

According to literature, failure in response to systemic therapy is associated with a worse prognosis in TNBC and our study corroborates this. To our knowledge there are no data regarding the role of neoadjuvant radiotherapy as an independent prognostic marker for disease recurrence.

In TNBC, the association between tumor size and nodal status is not as linear as in other breast cancer subtypes $[15,22]$. Since the pathologic nodal status is well established as one of the strongest prognostic factors in breast cancer, it was expected to show its prognostic value in our study. However, some studies did not confirm the prognostic significance of the nodal status in TNBC $[22,23]$.

In TNBC, it has been reported that there is a strong correlation with BRCA mutations According to the literature, approximately two out of nine women with TNBC harbor BRCA1 mutation [24]. However, several studies have investigated prognosis among BRCA mutation carriers and noncarriers and the prognostic impact on outcomes has not been definitely determined $[16,24]$. In our study, there is a low number of patients with BRCA mutation which can explain the lack of impact on prognosis. BRCA mutation testing is performed in patients $\leq 60$ years old with TNBC, which explains the significant number of patients with an unknown BRCA mutation status. 
One of the main goals of neoadjuvant chemotherapy, by allowing the downstaging of the tumor, is to convert candidates for mastectomy to breast-conserving surgery. In our study the percentage of mastectomy was far superior relative to breast conserving surgery, possibly related to the elevated percentage of advanced disease at presentation, the uncertainty about patterns of tumor shrinkage and temptation to excise the original tumor footprint and patient preference or contraindication for adjuvant radiotherapy, as well. Nevertheless, with the improvement of neoadjuvant chemotherapy with increasingly effective regimens, we hope to witness an increase in breast conserving surgery in TNBC.

This study has some limitations such as the retrospective methodology and missing data in patient's records. Nonetheless, the pCR rate in our study was higher $(48.8 \%)$ when compared to the data reported in literature (the CTNeoBC pooled analysis reported a pCR rate of 33.6 percent in triple negative breast cancer [14]). The study was also conducted in a relatively small number of patients (127) with a relatively short follow-up time of 29 months. Nonetheless, it is a real-life cohort from a resource-limited hospital.

\section{Conclusion}

In conclusion, TNBC represents a heterogeneous disease with different biological and clinical behavior. In this retrospective analysis of real-life non-metastatic TNBC, we were able to confirm the aggressiveness of this disease and identify a high-risk subgroup of patients for early disease recurrence. Although there are well-established prognostic factors with impact on DFS, in our study NART (performed due to refractory tumor response to neoadjuvant chemotherapy or significant toxicity) and pCR impacted as independent prognostic factors for relapse. Given the limited options after insufficient response to systemic therapy, radiotherapy could be a resource for downsizing, however, in our cohort it negatively impacted DFS. Despite being a highly heterogeneous disease, $\mathrm{pCR}$ is a well-established prognostic factor in TNBC and our study confirmed these results.

These results reinforce that this high-risk subgroup of patients might merit a closer follow-up. New biomarkers are mandatory for a better strati- fication of this heterogeneous population and there persists a medical need for more efficacious systemic treatments that improve response.

\section{Conflicts of interest}

None to declare.

\section{Funding}

This research did not receive any specific grant from funding agencies in the public, commercial, or not-for-profit sectors.

\section{Ethical approval}

The study was reviewed and approved by the Ethics Committee of the Portuguese Oncology Institute of Coimbra Francisco Gentil and a waiver of written informed consent from patients was granted considering the retrospective nature of the study.

\section{Authors' contribution}

Study concept and design: R.F.S.; data collection: R.F.S., A.R.M., F.M., T.C.P.; analysis and interpretation of data: R.F.S., A.R.G., J.C.C., A.P., M.M., P.M., S.P., S.B., T.C., I.P., G.S.; manuscript writing: R.F.S., A.R.G., J.C.C., A.P., M.M., P.M., S.P., S.B., T.C., I.P., G.S.; approval of final manuscript: all authors.

\section{References}

1. Swain S. Triple-Negative Breast Cancer: Metastatic Risk and Role of Platinum Agents 2008 ASCO Clinical Science Symposium, 2008.

2. The Global Cancer Observatory. https://gco.iarc.fr/.

3. Lin NU, Vanderplas A, Hughes ME, et al. Clinicopathologic features, patterns of recurrence, and survival among women with triple-negative breast cancer in the National Comprehensive Cancer Network. Cancer. 2012; 118(22): 5463-5472, doi: $10.1002 /$ cncr.27581, indexed in Pubmed: 22544643.

4. Hammond ME, Hayes DF, Dowsett M, et al. American Society of Clinical Oncology/College Of American Pathologists guideline recommendations for immunohistochemical testing of estrogen and progesterone receptors in breast cancer. J Clin Oncol. 2010; 28(16): 2784-2795, doi: 10.1200/JCO.2009.25.6529, indexed in Pubmed: 20404251.

5. Weigelt B, Reis-Filho J. Histological and molecular types of breast cancer: is there a unifying taxonomy? Nat Rev Clin Oncol. 2009; 6(12): 718-730, doi: 10.1038/ nrclinonc.2009.166, indexed in Pubmed: 19942925.

6. Dent R, Trudeau M, Pritchard KI, et al. Triple-negative breast cancer: clinical features and patterns of recurrence. Clin Cancer Res. 2007; 13(15 Pt 1): 4429-4434, doi: 10.1158/1078-0432.CCR-06-3045, indexed in Pubmed: 17671126. 
7. Cheang MCU, Voduc D, Bajdik C, et al. Basal-like breast cancer defined by five biomarkers has superior prognostic value than triple-negative phenotype. Clin Cancer Res. 2008; 14(5): 1368-1376, doi: 10.1158/1078-0432.CCR-071658, indexed in Pubmed: 18316557.

8. Hernandez-Aya LF, Chavez-Macgregor M, Lei X, et al. Nodal status and clinical outcomes in a large cohort of patients with triple-negative breast cancer. J Clin Oncol. 2011;29(19): 2628-2634, doi: 10.1200/JCO.2010.32.1877, indexed in Pubmed: 21606433.

9. Sørlie T, Perou CM, Tibshirani R, et al. Gene expression patterns of breast carcinomas distinguish tumor subclasses with clinical implications. Proc Natl Acad Sci U S A. 2001; 98(19): 10869-10874, doi: 10.1073/pnas.191367098, indexed in Pubmed: 11553815.

10. Kennecke H, Yerushalmi R, Woods R, et al. Metastatic behavior of breast cancer subtypes. J Clin Oncol. 2010; 28(20): 3271-3277, doi: 10.1200/JCO.2009.25.9820, indexed in Pubmed: 20498394.

11. Thike AA, lqbal J, Cheok PY, et al. Triple negative breast cancer: outcome correlation with immunohistochemical detection of basal markers. Am J Surg Pathol. 2010; 34(7): 956-964, doi: 10.1097/PAS.0b013e3181e02f45, indexed in Pubmed: 20495445.

12. Dent $R$, Hanna WM, Trudeau M, et al. Pattern of metastatic spread in triple-negative breast cancer. Breast Cancer Res Treat. 2009; 115(2): 423-428, doi: 10.1007/s10549-0080086-2, indexed in Pubmed: 18543098.

13. Liedtke C, Mazouni C, Hess KR, et al. Response to neoadjuvant therapy and long-term survival in patients with triple-negative breast cancer. J Clin Oncol. 2008; 26(8): 1275-1281, doi: 10.1200/JCO.2007.14.4147, indexed in Pubmed: 18250347.

14. Cortazar P, Zhang L, Untch M, et al. Pathological complete response and long-term clinical benefit in breast cancer: the CTNeoBC pooled analysis. Lancet. 2014; 384(9938): 164-172, doi: 10.1016/s0140-6736(13)62422-8, indexed in Pubmed: 24529560.

15. Yin L, Shuang H, Sheng C, et al. The Prognostic Value of Nodal Staging in Triple-Negative Breast Cancer - A Cohort from China. Sci Rep. 2018; 8(1): 9007, doi: 10.1038/s41598018-23999-8, indexed in Pubmed: 29899402.

16. Asaga S, Kinoshita T, Hojo T, et al. Prognostic factors for triple-negative breast cancer patients receiving preoperative systemic chemotherapy. Clin Breast Cancer. 2013; 13(1): 40-46, doi: 10.1016/j.clbc.2012.09.013, indexed in Pubmed: 23103363.
17. Wolff AC, Hammond ME, Hicks DG, et al. American Society of Clinical Oncology, College of American Pathologists. Recommendations for human epidermal growth factor receptor 2 testing in breast cancer: American Society of Clinical Oncology/College of American Pathologists clinical practice guideline update. J Clin Oncol. 2013; 31(31): 3997-4013, doi: 10.1200/JCO.2013.50.9984, indexed in Pubmed: 24101045.

18. von Minckwitz G, Untch M, Blohmer JU, et al. Definition and impact of pathologic complete response on prognosis after neoadjuvant chemotherapy in various intrinsic breast cancer subtypes. J Clin Oncol. 2012; 30(15): 1796-1804, doi: 10.1200/JCO.2011.38.8595, indexed in Pubmed: 22508812.

19. Fayanju OM, Ren Yi, Thomas SM, et al. The Clinical Significance of Breast-only and Node-only Pathologic Complete Response ( $\mathrm{pCR}$ ) After Neoadjuvant Chemotherapy (NACT): A Review of 20,000 Breast Cancer Patients in the National Cancer Data Base (NCDB). Ann Surg. 2018; 268(4): 591-601, doi: 10.1097/SLA.0000000000002953, indexed in Pubmed: 30048319.

20. Mladenovic J, Susnjar S, Tanic M, et al. Tumor response and patient outcome after preoperative radiotherapy in locally advanced noninflammatory breast cancer patients. J Buon. 2017; 22(2): 325-33, indexed in Pubmed: 28534352.

21. Coelho RC, Da Silva FML, Do Carmo IML, et al. Is there a role for salvage radiotherapy in locally advanced breast cancer refractory to neoadjuvant chemotherapy? Breast. 2017; 31: 192-196, doi: 10.1016/j.breast.2016.10.026, indexed in Pubmed: 27889595.

22. Foulkes WD, Grainge MJ, Rakha EA, et al. Tumor size is an unreliable predictor of prognosis in basal-like breast cancers and does not correlate closely with lymph node status. Breast Cancer Res Treat. 2009; 117(1): 199-204, doi: 10.1007/s10549-008-0102-6, indexed in Pubmed: 18600446.

23. Shibuta $K$, Ueo $H$, Furusawa $H$, et al. The relevance of intrinsic subtype to clinicopathological features and prognosis in 4,266 Japanese women with breast cancer. Breast Cancer. 2011; 18(4): 292-298, doi: 10.1007/s12282010-0209-6, indexed in Pubmed: 20571962.

24. Tun NM, Villani G, Ong $K$, et al. Risk of having BRCA1 mutation in high-risk women with triple-negative breast cancer: a meta-analysis. Clin Genet. 2014; 85(1): 43-48, doi: 10.1111/cge.12270, indexed in Pubmed: 24000781. 